\title{
THE NONTRIVIALITY OF THE RESTRICTION MAP IN THE COHOMOLOGY OF GROUPS
}

\author{
RICHARD G. SWAN ${ }^{1}$
}

An unpublished result ${ }^{2}$ of $B$. Mazur states that if $\pi$ is any nontrivial finite group then there is an $i>0$ such that $H^{i}(\pi, Z) \neq 0$. It is, course, trivial that $H^{i}(\pi, A) \neq 0$ for some $\pi$-module $A$. The point of Mazur's theorem is that we can even take $A=Z$, the ring of integers with trivial $\pi$-action. Mazur's proof of this theorem is geometric. It involves imbedding $\pi$ in a compact Lie group $G$ and studying the Leray-Cartan spectral sequence of the covering $G \rightarrow G / \pi$.

The purpose of this paper is to prove the following theorem which generalizes Mazur's result. ${ }^{3}$

TheOReM 1. Let $\pi$ be a finite group and $\rho$ a nontrivial subgroup of $\pi$. Then the restriction map $i(\rho, \pi): H^{i}(\pi, Z) \rightarrow H^{i}(\rho, Z)[2$, Chapter XII, $\S 8$ ] is nonzero for an infinite number of values of $i>0$.

As a consequence of this theorem, we get a generalization of Mazur's result.

CoRollary 1. Let $\pi$ be a finite group and let $p$ be a prime dividing the order of $\pi$. Then $H^{i}(\pi, Z)$ has a nonzero p-primary component for an infinite number of values of $i>0$.

To see this we have merely to use Theorem 1 , choosing for $\rho$ any nontrivial $p$-group in $\pi$.

The proof of Theorem 1 will also be geometric. In fact, I will actually prove the following much more general theorem whose proof must necessarily be geometric.

Theorem 2. Let $G$ be a compact, not necessarily connected Lie group. Let $H$ be a closed nontrivial subgroup of $G$, also not necessarily connected. Let $f: B_{H} \rightarrow B_{G}$ be the map of classifying spaces induced by the inclusion map $H \rightarrow G[1, \S 1]$. Then $f^{*}: H^{i}\left(B_{G}, Z\right) \rightarrow H^{i}\left(B_{H}, Z\right)$ is nonzero for an infinite number of values of $i$.

REMARK. If $H$ has an element of order $p$, the proof of this theorem will also show that $f^{*}: H^{i}\left(B_{G}, Z_{p}\right) \rightarrow H^{i}\left(B_{H}, Z_{p}\right)$ is nontrivial for an infinite number of values of $i$. If $H$ is infinite, it will show that

Received by the editors, November 24, 1959.

${ }^{1}$ Sponsored by the Office of Ordnance Research, U. S. Army under contract DA-11-022-ORD-2911.

2 I would like to thank W. Browder for communicating this result to me.

- This theorem was suggested to me by a problem of J. T. Tate. 
$f^{*}: H^{i}\left(B_{G}, Q\right) \rightarrow H^{i}\left(B_{H}, Q\right)$ is nonzero for an infinite number of values of $i$. Here $Q$ is the field of rational numbers.

Proof. By the Peter-Weyl theorem $G$ has a faithful unitary representation [4, Chapter VI, Theorem 4] and so can be imbedded in a unitary group $U(n)$. Also, $H$ has a subgroup isomorphic to $Z_{p}$ for some prime $p$. This is trivial if $H$ is finite, but if $H$ is infinite it contains a torus [3, Exposé 23, Theorem 1] which clearly has a subgroup isomorphic to $Z_{p}$. Since the map $B_{Z_{p}} \rightarrow B_{U(n)}$ factors through $f$, it will be sufficient to prove the theorem for the case $H \approx Z_{p}$ and $G \approx U(l)$. (If $H$ is infinite and we are trying to show that $H^{i}\left(B_{G}, Q\right.$ ) $\rightarrow H^{i}\left(B_{H}, Q\right)$ is nontrivial, it will suffice to consider the case where $G \approx U(n)$ and $H$ is a circle group. The rest of the proof will be substantially the same.)

Assume then that $H \approx Z_{p}, G \approx U(l)$. Imbed $H$ in a maximal torus $T$ of $G$. This can be done by taking any maximal torus $T$ containing a generator of $H$ [3, Exposé 23, Theorem 1]. Now, $H^{*}\left(B_{T}, Z\right)$ is a polynomial ring over $Z$ with generators $t_{1}, \cdots, t_{l} \in H^{2}\left(B_{T}, Z\right)$. The image of $H^{*}\left(B_{G}, Z\right)$ in $H^{2}\left(B_{T}, Z\right)$ consists of all symmetric polynomials in $t_{1}, \cdots, t_{l}[1, \S 4]$. Therefore to prove the theorem it will be sufficient to find sufficiently many symmetric polynomials which map nontrivially into $H^{*}\left(B_{H}, Z\right)$ under the map $g^{*}$ induced by $g: B_{H} \rightarrow B_{T}$. This map $g$ is, of course, induced by the inclusion $H \rightarrow T$.

Now, $H^{*}\left(B_{H}, Z\right)$ is a polynomial ring over $Z_{p}$ with a single generotor $\alpha \in H^{2}\left(B_{H}, Z\right)$ [2, Chapter XII, §7]. Therefore $g^{*}\left(t_{v}\right)=r_{\nu} \alpha$ with $r_{\nu} \in Z_{p}$. I claim that at least one $r_{\nu} \neq 0$. Suppose to the contrary that all $r_{\nu}=0$. Then $g^{*}: H^{2}\left(B_{T}, Z\right) \rightarrow H^{2}\left(B_{H}, Z\right)$ must be zero. Now $g: B_{H} \rightarrow B_{T}$ is a fiber map with fiber $T / H[1, \S 1]$. Of course, $T / H$ is a torus, being a connected abelian Lie group. The map $g^{*}: H^{2}\left(B_{T}, Z\right)$ $\rightarrow H^{2}\left(B_{H}, Z\right)$ is just the map $E_{2}^{2,0} \rightarrow E_{\infty}^{2,0}$ in the spectral sequence of this fibration. If it is zero, all elements of $E_{2}^{2,0}$ must bound. Therefore $d_{2}: E_{2}^{0,1} \rightarrow E_{2}^{2,0}$ must be onto. This shows that $T / H$ has rank $l$ and that $H_{1}(T / H, Z)=E_{2}^{0,1}$ has a base $\left\{x_{\nu}\right\}$ such that $d_{2} x_{\nu}=t_{\nu}$. (Of course it is trivial that $T / H$ has rank $l, H$ being finite, but I have arranged the proof so that it works for $H=S^{1}$ without essential change.) Now $E_{2}^{0,2}=H^{2}(T / H, Z)$ has a base $x_{\mu} x_{\nu}$ with $\mu<\nu$. Since $d_{2}$ is a derivation, $d_{2}\left(x_{\mu} x_{\nu}\right)=t_{\mu} \otimes x_{\nu}-t_{\nu} \otimes x_{\mu}$ in $E_{2}^{2,1}=H^{2}\left(B_{T}\right) \otimes H^{1}(T / H)$. Since these elements are linearly independent in $E_{2}^{2,1}, d_{2}$ is a monomorphism on $E_{2}^{0,2}$ and so $E_{3}^{0,2}=0$. Also $E_{3}^{2,0}=0$ and $E_{2}^{1,1}=0$. Thus the spectral sequence shows that $H^{2}\left(B_{H}, Z\right)=0$ which is absurd.

Now let $s$ be the number of indices $\nu$ for which $r_{\nu} \neq 0$. By renumbering we can assume that $r_{\nu} \neq 0$ for $\nu=1,2, \cdots, s$ and $r_{\nu}=0$ for $\nu>s$. 
Let $x$ be the $s$ th elementary symmetric function in $t_{1}, \cdots, t_{l}$. Then, for $k>0$,

$$
g^{*}\left(x^{k}\right)=\left(\prod_{1}^{s} r_{\nu}\right)^{k} \alpha^{\varepsilon k} \neq 0
$$

Since the $x^{k}$ are symmetric polynomials and have arbitrarily large dimensions, this proves the theorem.

REMARK. If $l$ is the smallest dimension of a faithful representation of $G$ over the complex numbers, the proof shows that $f^{*}: H^{i}\left(B_{G}, Z\right)$ $\rightarrow H^{i}\left(B_{H}, Z\right)$ is nonzero for some $i \leqq 2 l$ (since $i=2 s$ and $s \leqq l$ ). This is a best possible result if no further conditions are placed on $G, H$ and $l$. To see this for finite groups, let $H$ be the cyclic group of order $p$ permuting $p$ symbols and let $G$ be the normalizer of $H$ in the symmetric group $S_{p}$.

If $R$ denotes the real numbers, duality shows that $f_{*}: H_{i}\left(B_{H}, R / Z\right)$ $\rightarrow H_{i}\left(B_{G}, R / Z\right)$ is nonzero for an infinite number of values of $i$. But, if $\pi$ is finite, $H_{i}(\pi, R / Z) \approx H_{i-1}(\pi, Z)$, cf. [2, Chapter XII, Proof of Theorem 6.6]. Therefore Theorem 1 has the following corollary.

COROLlary 2. Let $\pi$ be a finite group and $\rho$ a nontrivial subgroup of $\pi$. Then the induced map $H_{i}(\rho, Z) \rightarrow H_{i}(\pi, Z)$ is nontrivial for an infinite number of values of $i>0$.

Equivalently, we may say that the transfer $t(\pi, \rho): \hat{H}^{i}(\rho, Z)$ $\rightarrow \hat{H}^{i}(\pi, Z)$ is nonzero for an infinite number of negative values of $i$ [2, Chapter XII, Exercise 8].

Note that the example $Z_{p} \subset Z_{p}+Z_{p}$ shows that the restriction map can be zero in all negative dimensions and the transfer zero in all positive dimensions.

It would be interesting to have a purely algebraic proof of Theorem 1 but I know of no such proof.

\section{REFERENCES}

1. A. Borel et J.-P. Serre, Groupes de Lie et puissances réduites de Steenrod, Amer. J. Math. vol. 75 (1953) pp. 409-448.

2. H. Cartan and S. Eilenberg, Homological algebra, Princeton, 1956.

3. P. Cartier, Séminaire Sophus Lie, Ecole Normale Supérieure, Paris, 1954-1955.

4. C. Chevalley, Theory of Lie groups, Princeton, 1946.

The University of Chicago 